\title{
Interaction Laws in Viscous-Inviscid Coupling
}

\author{
Arthur E. P. Veldman ${ }^{1}$ and Edith G.M. Coenen ${ }^{2}$ \\ 1 University of Groningen, Institute of Mathematics and Computing Science \\ (http://www.math.rug.nl/ veldman/) \\ 2 Present address: KPN, Den Haag
}

Summary. Viscous-inviscid coupling methods for the computation of aerodynamic boundary layers are discussed, with emphasis on the quasi-simultaneous method. Its interaction law is analysed using matrix theory and reduced to its essentials. The redesigned interaction law is tested for separated airfoil flow at maximum lift.

\section{Introduction}

The accurate and fast prediction of viscous flow over two- and three-dimensional surfaces is an important problem in aero- and hydrodynamics. The continuing advances in efficiency of numerical algorithms, together with the increasing speed and memory size of computers, are enabling viscous flows to be calculated by methods that solve the full (Reynolds-averaged) Navier-Stokes equations. Whilst Navier-Stokes simulation potentially offers generality, its computational requirements currently limit its use for practical applications, especially within a design optimisation environment.

An alternative is to use the older technique of viscous-inviscid interaction (VII), where an inviscid-flow solver is coupled to a viscous boundary-layer calculation method (Figure 1). VII methods have shown to be very efficient and robust (Lock and Williams [1987]). For many cases of aerodynamic interest the coupled solution matches experimental data as well as Navier-Stokes simulation, and this at much lower computational cost.

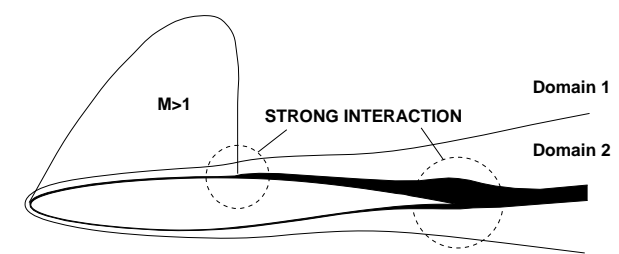

Fig. 1. Decomposition of flow field into boundary layer and inviscid flow 
Since Prandtl [1905] introduced his boundary-layer concept in 1904, several VII methods have been developed. The oldest technique is known as the direct method, which solves the boundary layer with a prescribed velocity (pressure) distribution $u_{e}$. In turn, the boundary layer expresses its presence in terms of a virtually thickened profile, called displacement thickness $\delta^{*}$.

For situations with attached flow the direct method works well. However, when regions of reversed flow are present the direct boundary-layer calculation breaks down. In 1948, Goldstein [1948] presented an extensive study of the breakdown, but no definitive conclusion about its origin could be given. Since then the singularity at flow separation bears his name.

It was not until 1966 that the first clue on how to prevent this singularity was given, when Catherall and Mangler [1966] presented calculations of a boundary layer with prescribed $\delta^{*}$. They were able to pass the critical point of flow separation, but ran into numerical difficulties somewhat further on in the reversed-flow region. A further clue was provided by the asymptotic tripledeck theory introduced by Stewartson [1969], indicating a change in hierarchy between the inviscid-flow region and the boundary layer.

Led by these ideas, in the late 70's alternatives for the direct VII method were introduced. An obvious choice is to reverse the order of information exchange. This inverse method survives in flow separation, but its convergence is extremely slow. Henceforth, both methods were mixed into the semi-inverse method (LeBalleur [1978]), where both flow regions are solved with prescribed $\delta^{*}$, which is then updated based on the difference in the respective $u_{e}$ distributions. The latter update requires careful tuning of relaxation parameters.

Another idea was to avoid any hierarchy in the treatment of both flow regions. However, a fully simultaneous approach would require both sets of flow equations to be merged into one big system, which is quite complicated in software terms and defies any flexibility in flow modeling. Hence, an attempt was made to approximate such a simultaneous approach. Thus the concept of the interaction law came up: a simple, yet powerful, description of the inviscid flow, which can easily be solved simultaneously with the boundarylayer equations (Veldman [1981]). In this paper we will analyse this quasisimultaneous approach and simplify the interaction law. We end up with a method which is very close to the direct method, and yet has no problems with reversed flow, as demonstrated with a calculation of airfoil flow.

\section{Quasi-Simultaneous VII and the Interaction Law}

In an abstract setting, the coupled VII problem can be written as

$$
\left.\begin{array}{ll}
\text { external inviscid flow: } & u_{e}=E \delta^{*} \\
\text { boundary-layer flow: } & u_{e}=-V \delta^{*}
\end{array}\right\} \Rightarrow(V+E) \delta^{*}=0 .
$$

The classical way of solving these equations is to prescribe $\delta^{*}$ to the external inviscid flow, and then to return $u_{e}$ to the boundary layer. As the boundary- 
layer step breaks down when flow separation occurs, the quasi-simultaneous method tries to avoid the iterative hierarchy involved. The basic idea is to inform the boundary layer instantaneously how the external flow will react on changes inside the boundary layer. Hereto, a sufficiently accurate, yet simple, approximation of the external inviscid flow is introduced, denoted as $u_{e}=I \delta^{*}$. This interaction law is to be solved simultaneously with the boundary-layer equations, i.e.

$$
\left.\begin{array}{rl}
u_{e}^{(n)}-I \delta^{*(n)} & =(E-I) \delta^{*(n-1)} \\
u_{e}^{(n)}+V \delta^{*(n)} & =0
\end{array}\right\} \Rightarrow(V+I) \delta^{*(n)}=(I-E) \delta^{*(n-1)} .
$$

Note that the VII iterations 'only' need to account for the difference between the external flow $E$ and its approximation $I$.

Next the question arises how to choose the interaction law. A fair description of how an inviscid flow reacts on displacement effects is delivered by thin-airfoil theory, in its simplest form given by

$$
u_{e}(x)=u_{e 0}(x)+\frac{1}{\pi} \int_{\Gamma} \frac{\mathrm{d} \delta^{*}}{\mathrm{~d} \xi} \frac{\mathrm{d} \xi}{x-\xi},
$$

where $u_{e 0}$ is the edge velocity without displacement effects. Also triple-deck theory provides this type of approximation, which makes (3) a good candidate as an interaction law. In fact, this interaction law (describing thickness effects) together with its anti-symmetric counterpart (describing camber effects) has been used successfully in subsonic and transonic(!) airfoil/wake calculations (Veldman et al. [1990]). As these thin-airfoil expressions are somewhat complicated it is worthwhile to try to simplify the interaction law, yet retaining a robust and efficient VII algorithm. Thus, the question to be answered is

$$
\text { How 'small' can I be chosen? }
$$

As a reminder, the direct choice $I=0$ blows up in Goldstein's singularity. We will first address this question from a theoretical point of view. Thereafter, the usefulness of the theory will be demonstrated on realistic flow problems.

\section{A Model Problem}

As a model problem to shape the theory, the flow past an indented plate (Figure 2) is studied for which the external flow will be described by the thinairfoil expression (3). It is our aim to construct a simple interaction law for this case. Let us first collect some properties of the operators $E$ and $V$.

External Flow. The integral (3) is discretized on a uniform grid with mesh size $h$. The displacement thickness $\delta^{*}$ is interpolated by a piece-wise linear function; only on the two intervals adjacent to the Cauchy principal value a quadratic is used: 


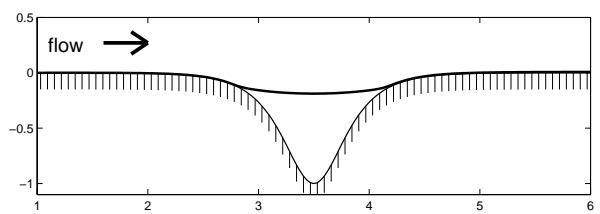

Fig. 2. Geometry sketch of indented plate geometry

$$
\begin{aligned}
\frac{1}{\pi} \int_{\Gamma} \frac{\mathrm{d} \delta^{*}}{\mathrm{~d} \xi} \frac{\mathrm{d} \xi}{x_{i}-\xi} & =\frac{1}{\pi}\left\{\int_{x_{i-1}}^{x_{i+1}}+\Sigma_{j \neq i-1, i} \frac{1}{\pi} \int_{x_{j}}^{x_{j+1}}\right\} \frac{\mathrm{d} \delta^{*}}{\mathrm{~d} \xi} \frac{\mathrm{d} \xi}{x_{i}-\xi} \\
& \approx-\left.\frac{2 h}{\pi} \frac{\mathrm{d}^{2} \delta^{*}}{\mathrm{~d} \xi^{2}}\right|_{i}+\left.\frac{h}{\pi} \sum_{j \neq i-1, i} \frac{\mathrm{d} \delta^{*}}{\mathrm{~d} \xi}\right|_{j+1 / 2} \ln \left|\frac{i-j}{i-j-1}\right|
\end{aligned}
$$

The corresponding discrete matrix $\mathbf{E}$ is symmetric, positive definite with diagonal $4 / \pi h$, and with non-positive off-diagonal entries.

Boundary-Layer Flow. Referring e.g. to Veldman [1984], a discrete boundarylayer operator typically is lower diagonal, with positive diagonal entries for attached flow and (slightly) negative diagonal entries for reversed flow.

\section{Theory of Viscous-Inviscid Interaction}

Throughout our mathematical analysis the following assumption is made:

Assumption The matrix $\mathbf{V}+\mathbf{E}$ is assumed to be an M-matrix, i.e. it has positive diagonal entries, non-positive off-diagonal entries and all eigenvalues have positive real part.

The location of the eigenvalues corresponds with steady flow; the sign of the matrix entries allows theory, and will hold approximately in practice.

VII Iterations. An interaction law (2) corresponds with a splitting $\mathbf{V}+\mathbf{E}=$ $(\mathbf{V}+\mathbf{I})-(\mathbf{I}-\mathbf{E})$. When $\mathbf{I} \geq \mathbf{E}$ and when also $\mathbf{V}+\mathbf{I}$ is an M-matrix, then $(\mathbf{V}+\mathbf{E})^{-1} \geq(\mathbf{V}+\mathbf{I})^{-1} \geq 0$ [Horn and Johnson, 1991, p. $117 \& 127$ ] and the splitting is regular [Varga, 1962, p. 88]. As the off-diagonals of $\mathbf{E}$ are non-positive, this suggests to construct $\mathbf{I}$ from $\mathbf{E}$ by dropping one or more offdiagonals. Subsequently, the comparison theorem on regular splittings [Varga, 1962 , p. 90] implies that the convergence of the VII iterations deteriorates monotonously with the number of dropped off-diagonals in $\mathbf{I}$.

Boundary-Layer Iterations. In each VII iteration a boundary-layer computation has to be performed, in which (2) is to be solved. This is done by repeated marching through the boundary layer, starting near the stagnation point and proceeding in downstream direction. Thus, a Gauss-Seidel type of iteration is performed. This method 'only' has to iterate on the upper triangular part of the matrix $\mathbf{V}+\mathbf{I}$, which here consists of entries from $\mathbf{I}$. Hence 
it may be expected that a 'small' upper-diagonal part will speed up convergence. And, indeed, under the above assumption it can be proven that the Gauss-Seidel convergence improves monotonously with the number of dropped off-diagonals in I (Coenen [2001]). This is opposite to the behaviour of the VII iterations, hence a trade-off is opportune (see below). Further, it is remarked that an interaction law which only consists of a main diagonal does not require boundary-layer iterations.

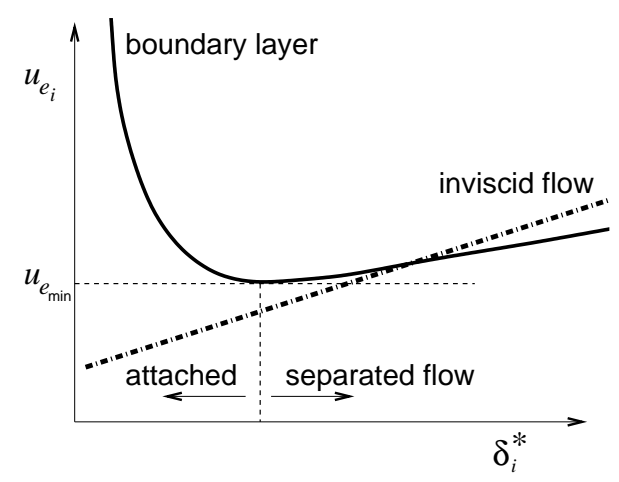

Fig. 3. A sketch of the boundary-layer behaviour, combined with an inviscid-flow relation

Robustness. The boundary-layer formulation is highly nonlinear. A sketch of the situation is given in Figure 3. Here, at a fixed boundary-layer station, the dependence between the edge velocity and the displacement thickness is shown (Veldman [1984]). This sketch shows clearly that below a certain value for a prescribed $u_{e}$ no solution can be found anymore; this non-existence of a boundary-layer solution induces the breakdown! Prescribing a linear combination of $u_{e}$ and $\delta^{*}$, as is the case when an interaction law is applied, should be useful provided the coefficient of $\delta^{*}$ stays away from zero sufficiently. Also during the iterations the process should not break down, hence the eigenvalues of the interaction matrix $\mathbf{I}$, and herewith the eigenvalues of $\mathbf{V}+\mathbf{I}$, should stay sufficiently far from the imaginary axis.

Again theory can be developed. With the above assumption, $\mathbf{V}+\mathbf{I}$ can be written as a constant positive diagonal matrix minus a non-negative matrix. For the latter type of matrices the largest eigenvalue grows monotonously with the matrix entries (the Perron-Frobenius theorem [Varga, 1962, p. 30]). Thus for $\mathbf{V}+\mathbf{I}$ this dependency holds for the smallest eigenvalue. With $\mathbf{I} \geq \mathbf{E}$, this eigenvalue is located in the stable (positive) half plane. Further, it grows with the number of dropped diagonals, herewith increasing the robustness of the boundary-layer calculation. Also in this respect an interaction law consisting of only the main diagonal of $\mathbf{E}$ scores best. 


\section{Viscous-Inviscid Interaction in Practice}

\section{Indented Plate}

The applicability of the theory will first be investigated with the above indented plate model. The length of the domain is five units, with a dent up to one unit deep (which is very deep in comparison with the boundary-layer thickness, but gives a severe testcase for the VII algorithm). The Reynolds number based on unit length is $10^{8}$. The boundary layer is modelled with Head's entrainment method; see Coenen [2001].

The interaction law $\mathbf{I}$ is chosen by simply dropping off-diagonals in the 'exact' inviscid flow matrix E. Figure 4 gives the number of VII iterations as a function of the number of retained diagonals (including the main diagonal). Three flow situations have been distinguished: one with attached flow (when the dent is very shallow), one with mild separation, and one with severe separation (as in Figure 2).

For all choices of I the VII iterations are found to converge. Moreover, according to theory, the convergence of the VII iterations improves monotonously with the number of diagonals retained in $\mathbf{I}$. The limit number of iterations is 2-3. For attached flow this can be compared with the direct method which also requires 3 iterations to converge (in the separated-flow cases the direct method breaks down).
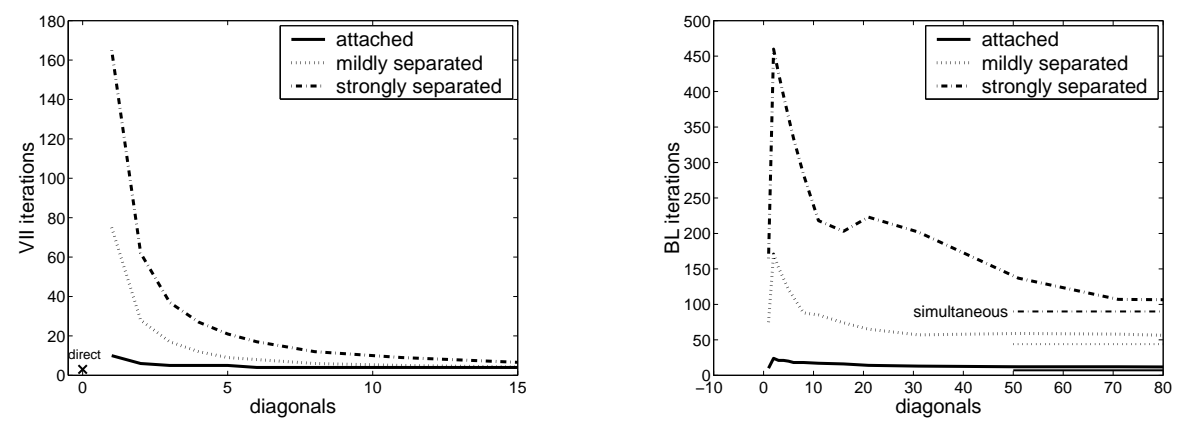

Fig. 4. Number of VII iterations (left) and total number of boundary-layer sweeps (right) as a function of the number of retained diagonals

Further, the number of VII iterations drops fast when the number of retained diagonals increases. On the other hand this leads to slower convergence of the boundary-layer iterations, and therefore the total number of boundarylayer sweeps has also been monitored in Figure 4. A local minimum exists when the interaction law only consists of the main diagonal; remember that in this case only one boundary-layer sweep per VII iteration is required. When one off-diagonal is added, the number of Gauss-Seidel sweeps per VII iteration increases, such that the total number of boundary-layer sweeps also 
increases, even though the number of VII iterations has decreased. Adding more off-diagonals the decrease in VII iterations becomes dominant. A minimum number of boundary-layer sweeps is found in the limit $\mathbf{I}=\mathbf{E}$. Here, the number of boundary-layer sweeps should be equal to the number required for a fully simultaneous treatment, i.e. when the system (1) is solved by GaussSeidel. Indeed, this is found to be the case.

Thus for the interaction law two interesting choices exist. One option is to choose it according to the 'full' external flow; the other is to choose it equal to only the diagonal $4 / \pi h$ of the external-flow matrix. As the first option is against our quest for simplicity, below we will test the second option on a realistic problem of boundary-layer flow past a two-dimensional airfoil.

\section{Subsonic Airfoil Flow}

The above ideas on simplifying the interaction law will now be tested for aerodynamic flow past a NACA0012 airfoil (at $R e=9 \cdot 10^{6}$, and $M_{\infty}=0$ ); experimental data is available. The inviscid flow is modelled by potential theory, and computed by means of a panel method. Boundary layer plus wake are modelled with Head's entrainment method (for more information we refer to Coenen [2001]). They are solved together with the interaction law $\mathbf{I}=\operatorname{diag}(4 / \pi h)$. We stress that this interaction law is unaware of the Kutta condition and its effect on the global circulation; it only accounts for the local VII physics but this turns out to be sufficient.

A large part of the lift polar has been computed. The calculations turn out to be highly robust. It appears that even for separated-flow cases beyond maximum lift the calculations converge without any need for a good initial guess; they can be started from scratch. The number of VII iterations typically is less than 100 at zero lift upto 1000 around maximum lift (computing times count in seconds on an average PC). Only for larger angles of attack the computations break down; using a good initial guess obtained at a slightly smaller angle does not help.

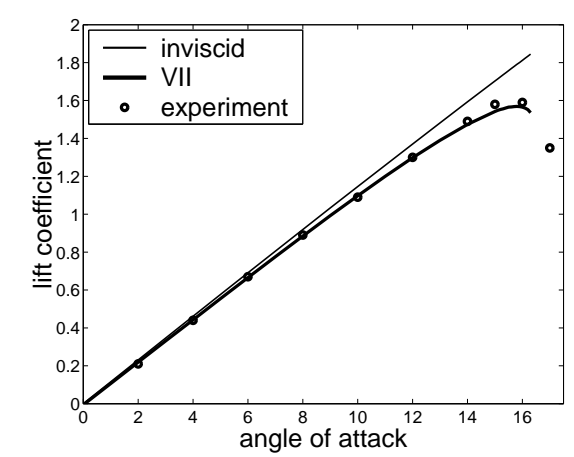

Fig. 5. Lift polar for NACA0012 airfoil: calculation versus experiment 


\section{Conclusion}

A short overview of viscous-inviscid interaction has been presented, starting with Prandtl one century ago, and encountering Goldstein's analysis half a century later. Today, after a whole century of boundary-layer research, it has been found that Goldstein's singularity can be prevented by changing the 'classical' boundary condition of prescribed edge velocity into

$$
\left(u_{e}+\frac{4}{\pi h} \delta^{*}\right)^{(\text {new })}=\left(u_{e}+\frac{4}{\pi h} \delta^{*}\right)^{(\text {old })}
$$

This slight change, unlikely to be further simplified, results in a highly robust calculation method, applicable to airfoil calculations beyond maximum lift.

\section{References}

D. Catherall and K. Mangler. The integration of the two-dimensional laminar boundary-layer equations past the point of vanishing skin friction. J. Fluid Mech., 26:163-182, 1966.

E. Coenen. Viscous-Inviscid Interaction with the Quasi-Simultaneous Method for 2D and 3D Airfoil Flow. PhD thesis, Groningen, 2001.

S. Goldstein. On laminar boundary layer flow near a point of separation. Quart. J. Mech. Appl. Math., 1:43-69, 1948.

R. Horn and C. Johnson. Topics in Matrix Analysis. CUP, 1991.

J. LeBalleur. Couplage visqueux-non visqueux: méthode numérique et applications aux écoulements bidimensionnels transsoniques et supersoniques. La Recherche Aérospatiale, 183:65-76, 1978.

R. Lock and B. Williams. Viscous-inviscid interactions in external aerodynamics. Prog. Aerospace Science, 24:51-171, 1987.

L. Prandtl. Ueber Fluessigkeitsbewegung mit kleiner Reibung. In Verhandlungen des dritten internationalen Mathematischen Kongresses, Heidelberg, pages 484-491, Leipzig, 1905. Teubner Verlag.

K. Stewartson. On the flow near the trailing edge of a flat plate II. Mathematika, 16:106-121, 1969.

R. Varga. Matrix Iterative Analysis. Prentice-Hall, 1962.

A. Veldman. New, quasi-simultaneous method to calculate interacting boundary layers. AIAA J., 19:79-85, 1981.

A. Veldman. A numerical view on strong viscous-inviscid interaction. In W. Habashi, editor, Computational Methods in Viscous Flows, pages 343363, Southampton, 1984. Pineridge Press.

A. Veldman, J. Lindhout, E. de Boer, and M. Somers. Vistrafs: a simulation method for strongly-interacting viscous transonic flow. In T. Cebeci, editor, Numerical and Physical Aspects of Aerodynamic Flow IV, pages 37-51, Berlin, 1990. Springer Verlag. 